\title{
Internal Barrier Layer Capacitance Effects in Neodymium Copper Tantalate Ceramics
}

\author{
D. SZWAGIERCZAK* \\ Institute of Electron Technology, Kraków Division, Zabłocie 39, 30-701 Kraków, Poland
}

\begin{abstract}
A new perovskite material $\mathrm{Nd}_{2 / 3} \mathrm{CuTa}_{4} \mathrm{O}_{12}$ was applied as a naturally formed internal barrier layer capacitor. The powder prepared by solid state synthesis and ball milling was pressed into pellets and sintered at $1180-1220^{\circ} \mathrm{C}$. Dielectric properties of ceramic samples were characterized by impedance spectroscopic studies carried out in the temperature range from -55 to $700^{\circ} \mathrm{C}$ at frequencies $10 \mathrm{~Hz} \div 2 \mathrm{MHz}$. Two types of the dielectric response were revealed - a high frequency response attributed to grains which occurred at low temperatures, and a low frequency one related to grain boundaries which dominated at higher temperatures. Resistances and capacitances of grains were found to be two orders lower than those of grain boundaries. Two plateaus were observed in the dielectric permittivity versus frequency plots - a low frequency step corresponding to a high value of $10^{4}$ and a high frequency step at a level of 40. Scanning electron microscopy observations and energy dispersive spectroscopy analysis confirmed that $\mathrm{Nd}_{2 / 3} \mathrm{CuTa}_{4} \mathrm{O}_{12}$ ceramics were composed of semiconducting grains and insulating oxygen-enriched grain boundaries. The formation of such an electrically heterogeneous system during the one step fabrication process in air leads to spontaneous internal barrier layer capacitance effects responsible for a high and relatively stable dielectric permittivity of the developed material.
\end{abstract}

PACS: 77.22.-d, 81.05.Je, 81.20.-n, 84.32.Tt, 84.37.+q

\section{Introduction}

Search for new materials with high dielectric constant is an important task in view of increasing demand for miniaturization and improved performance of capacitive elements. Since the last decade a group of nonferroelectric materials of the complex perovskite structure have attracted attention due to their high and stable dielectric permittivity. The most intensively studied representative of this group is $\mathrm{CaCu}_{3} \mathrm{Ti}_{4} \mathrm{O}_{12}$ [1-4]. Other members of this family are $\mathrm{Bi}_{2 / 3} \mathrm{Cu}_{3} \mathrm{Ti}_{4} \mathrm{O}_{12}[5,6], \mathrm{Cu}_{2} \mathrm{Ta}_{4} \mathrm{O}_{12}$ [7-9], $\mathrm{Dy}_{2 / 3} \mathrm{CuTa}_{4} \mathrm{O}_{12}$ [10]. Strong tilting of $\mathrm{TiO}_{6}$ octahedra and fourfold coordination of small $\mathrm{Cu}$ ions in $\mathrm{A}$ sites of the $\mathrm{ABO}_{3}$ perovskite structure are characteristic of these compounds [1]. In the $\mathrm{Cu}_{2} \mathrm{Ta}_{4} \mathrm{O}_{12}$ structure a half of A sites is unoccupied. Internal barrier layer (IBL) and/or surface barrier layer (SBL) capacitance effects have been indicated as origins of giant dielectric permittivity in the considered materials. Internal barrier layers can be formed by insulating grain boundaries of semiconducting grains or by planar defects. Surface barrier layers can be created at electrode-sample interfaces or at boundaries between the sample interior and surface layers with different compositions or defect structures.

In the present work, the results of impedance spectroscopic studies, microstructure investigations and EDS analysis are reported for a new material in this group $\mathrm{Nd}_{2 / 3} \mathrm{CuTa}_{4} \mathrm{O}_{12}$ ceramic with the $\mathrm{Cu}_{2} \mathrm{Ta}_{4} \mathrm{O}_{12}$ structure.

\footnotetext{
* e-mail: dszwagi@ite.waw.pl
}

\section{Experimental}

A new material of the composition $\mathrm{Nd}_{2 / 3} \mathrm{CuTa}_{4} \mathrm{O}_{12}$ was synthesized by a conventional solid state reaction at $1180^{\circ} \mathrm{C}$ for $25 \mathrm{~h}$. The product was ball milled for $8 \mathrm{~h}$, pressed into pellets and sintered at $1180-1220^{\circ} \mathrm{C}$ for 12-25 h. Phase composition of the material was examined by an X'Pert Philips diffractometer. Microstructure and chemical composition of the ceramics were investigated using a FEI scanning electron microscope and an EDAX Genesis EDS system. Dielectric properties of the samples were studied by impedance spectroscopy using a LCR QuadTech meter in the temperature range from -55 to $700^{\circ} \mathrm{C}$ at frequencies $10 \mathrm{~Hz} \div 2 \mathrm{MHz}$. Dc conductivity of $\mathrm{Nd}_{2 / 3} \mathrm{CuTa}_{4} \mathrm{O}_{12}$ ceramics was measured in the temperature range $20-700^{\circ} \mathrm{C}$.

\section{Results and discussion}

In Fig. 1a and b the frequency dependences of the real and the imaginary parts of impedance are depicted for $\mathrm{Nd}_{2 / 3} \mathrm{CuTa}_{4} \mathrm{O}_{12}$ ceramic sintered at $1220^{\circ} \mathrm{C}$. Figure 2 presents the complex plane impedance plots for a few selected temperatures in the range from -55 to $700{ }^{\circ} \mathrm{C}$. Three types of dielectric response can be distinguished on the basis of the impedance data. At temperatures above $100^{\circ} \mathrm{C}$, the predominant response is related to grain boundaries.

This response is reflected by a low frequency plateau in the $Z^{\prime}$ versus frequency dependence (Fig. 1a), by a main peak in the $Z^{\prime \prime}$ versus frequency dependence (Fig. 1b) and 


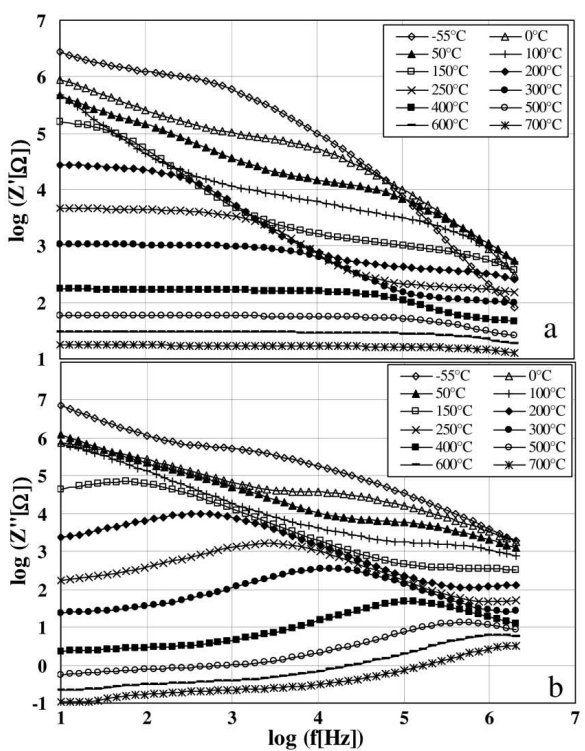

Fig. 1. Frequency dependence of the real (a) and the imaginary (b) parts of impedance in a temperature range from -55 to $700^{\circ} \mathrm{C}$ for $\mathrm{Nd}_{2 / 3} \mathrm{CuTa}_{4} \mathrm{O}_{12}$ ceramic sintered at $1220^{\circ} \mathrm{C}$.

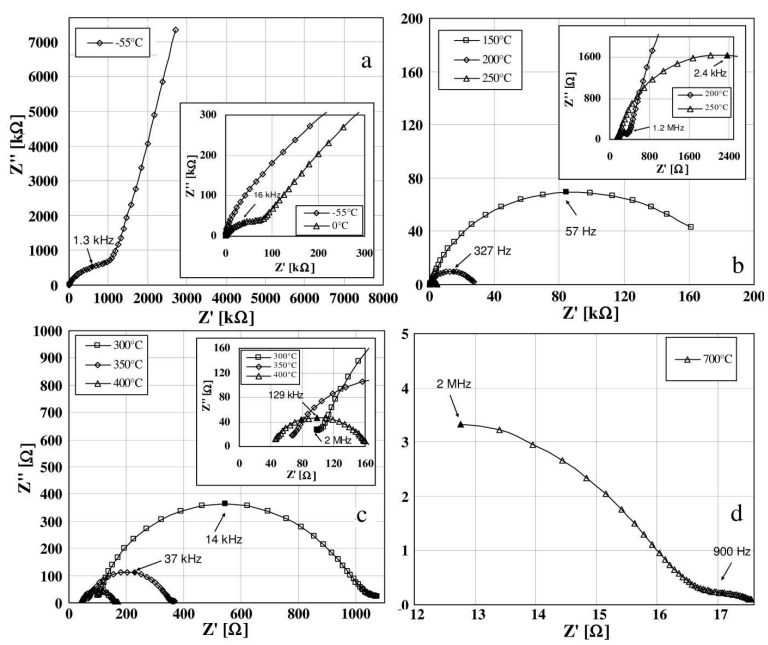

Fig. 2. The imaginary versus the real part of impedance for different temperatures in a range from -55 to $700^{\circ} \mathrm{C}$ for $\mathrm{Nd}_{2 / 3} \mathrm{CuTa}_{4} \mathrm{O}_{12}$ ceramic.

by a large arc in the complex impedance plot (Fig. 2b-d). At low temperatures, a second peak of $Z^{\prime \prime}$ (Fig. 1b) and a second smaller arc in the complex impedance plots (Fig. 2a) are observed at high frequency sides of the curves. This response is attributed to grains. At the highest investigated temperatures (above $600^{\circ} \mathrm{C}$ ) and at the lowest frequencies, a third flat $Z^{\prime \prime}$ maximum and an additional small arc in the $Z^{\prime \prime}=f\left(Z^{\prime}\right)$ plot occur which could be ascribed to the electrode-sample interface.

In Fig. 3a the resistances of grains and grain boundaries determined on the basis of diameters of relevant semicircles in the complex impedance plots are displayed

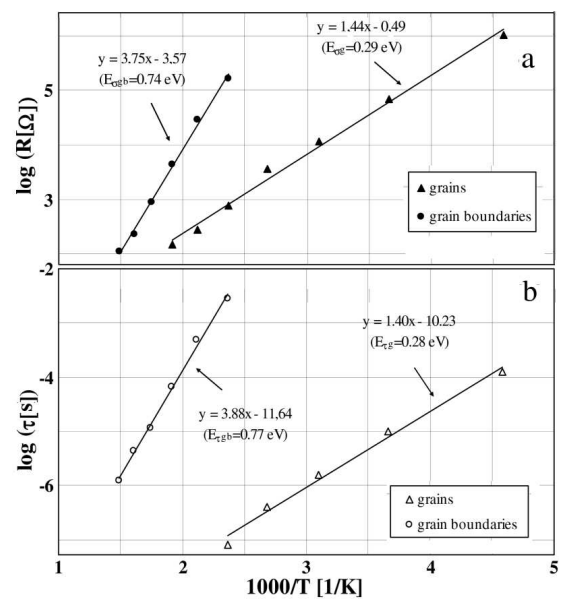

Fig. 3. Resistances (a) and relaxation times (b) for grains and grain boundaries of $\mathrm{Nd}_{2 / 3} \mathrm{CuTa}_{4} \mathrm{O}_{12}$ ceramic as a function of reciprocal temperature.

as a function of reciprocal temperature. Figure $3 \mathrm{~b}$ shows such temperature dependence of the relaxation times $\tau$ calculated from the relationship $2 \pi f_{\max } \tau=1\left(f_{\max }-\right.$ frequency corresponding to the maximum in the complex impedance plot). Both dependences follow well the Arrhenius law. The activation energies determined from the slopes of the plots are $0.29 \mathrm{eV}$ and $0.74 \mathrm{eV}$ for resistances of grains and grain boundaries, respectively. For relaxation times, the activation energies are $0.28 \mathrm{eV}$ and $0.77 \mathrm{eV}$ for grains and grain boundaries, respectively. The similarity of both types of activation energies implies that electrical conduction and dielectric relaxation processes are determined by the transport of the same charge carriers. Below $250^{\circ} \mathrm{C}$, the resistances of grain boundaries are more than one order higher than those of grains.

Figure $4 \mathrm{a}, \mathrm{b}$ presents in $\log -\log$ coordinates the frequency dependences of the real part of permittivity $\varepsilon^{\prime}$ and the imaginary part of electrical modulus $M^{\prime \prime}$ in a wide temperature range. In the $\varepsilon^{\prime}$ versus frequency plots there are two steps. The low frequency step corresponding to a high value of $10^{4}$ is attributed to grain boundary barrier layer capacitance. The high frequency step at a level of 45 which dominates at low temperatures is related to intrinsic properties of grains.

As shown in Fig. 4b, there are two sets of peaks in the $M^{\prime \prime}$ versus frequency curves in the temperature range from -55 to $350^{\circ} \mathrm{C}$. The higher peaks which appear at low temperatures are attributed to grains, whereas the lower humps observed at higher temperatures - to grain boundaries. The values of $M^{\prime \prime}$ maxima are inversely proportional to capacitances. Thus, the obtained electrical modulus values imply that capacitances of grains are two orders lower than those of grain boundaries, and both the capacitances are temperature independent.

In Fig. 5a the logarithm of dc electrical conductivity of the ceramic is depicted as a function of reciprocal temperature. The activation energies determined from 


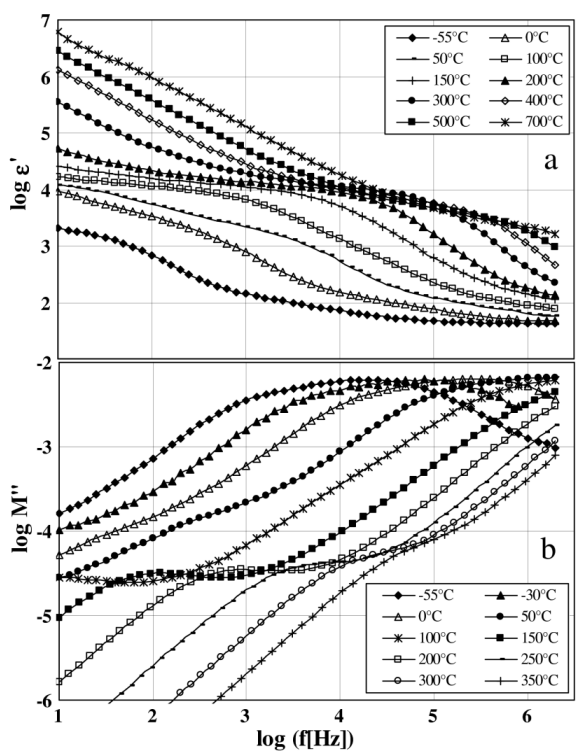

Fig. 4. Frequency dependence of the real part of permittivity (a) and the imaginary part of electric modulus (b) in a temperature range from -55 to $350-700^{\circ} \mathrm{C}$ for $\mathrm{Nd}_{2 / 3} \mathrm{CuTa}_{4} \mathrm{O}_{12}$ ceramic.

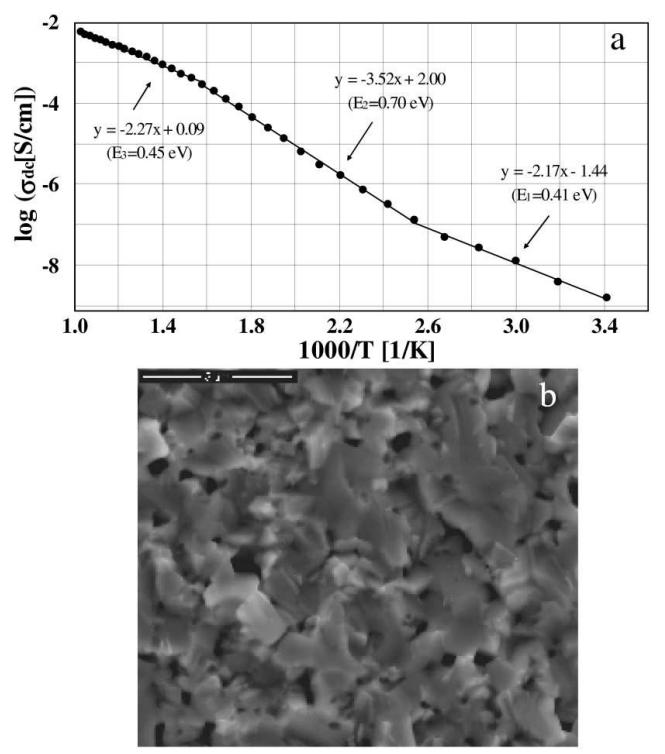

Fig. 5. (a) Logarithm of dc electrical conductivity as a function of reciprocal temperature, (b) SEM image of $\mathrm{Nd}_{2 / 3} \mathrm{CuTa}_{4} \mathrm{O}_{12}$ ceramic sintered at $1220^{\circ} \mathrm{C}$.

the slopes of linear parts of the plots are $0.41,0.70$ and $0.45 \mathrm{eV}$ in different temperature ranges. The activation energy of dc conduction in the temperature range 120$360^{\circ} \mathrm{C}(0.70 \mathrm{eV})$ is close to that of resistances of grain boundaries $(0.74 \mathrm{eV})$ determined from the impedance data.
Figure 5b illustrates a scanning electron microscopy (SEM) image of a fractured cross-section of $\mathrm{Nd}_{2 / 3} \mathrm{CuTa}_{4} \mathrm{O}_{12}$ ceramic sintered at $1220^{\circ} \mathrm{C}$. Irregular grain growth is observed leading to grain sizes ranging from 0.5 to $5 \mu \mathrm{m}$. The results obtained by energy dispersive spectroscopy (EDS) method indicate that the content of oxygen at grain boundaries is distinctly higher than that in grain interiors.

\section{Conclusions}

Impedance spectroscopic studies of $\mathrm{Nd}_{2 / 3} \mathrm{CuTa}_{4} \mathrm{O}_{12}$ ceramics sintered at $1180-1220^{\circ} \mathrm{C}$ reveal three types of the dielectric response which can be attributed to grains, grain boundaries and electrode-sample interfaces. At temperatures above $100^{\circ} \mathrm{C}$, the predominant response is related to grain boundaries. Resistances and capacitances of grains are two orders lower than those of grain boundaries. The EDS analysis indicates an oxygen enrichment at grain boundaries. The most plausible explanation of dielectric behavior of $\mathrm{Nd}_{2 / 3} \mathrm{CuTa}_{4} \mathrm{O}_{12}$ ceramic seems to be IBLC mechanism related to spontaneous formation of insulating grain boundaries between semiconducting grains.

\section{Acknowledgments}

The work has been financed by the Polish Ministry of Science and Higher Education under grant N N507 218240 .

\section{References}

[1] M.A. Subramanian, D. Li, N. Duan, B.A. Reisner, A.W. Sleight, J. Solid State Chem. 151, 323 (2000).

[2] D.C. Sinclair, T.B. Adams, F.D. Morrison, A.R. West, Appl. Phys. Lett. 80, 2153 (2002).

[3] B. Shri Prakash, K.B.R. Varma, J. Mater. Sci., Mater. Electron. 17, 899 (2006).

[4] M. Li, Z. Shen, M. Nygren, A. Feteira, D.C. Sinclair, A.R. West, J. Appl. Phys. 106, 104106 (2009).

[5] J. Liu, C.G. Duan, W.G. Yin, W.N. Mei, R.W. Smith, J.R. Hardy, Phys. Rev. B 70, 144106 (2004).

[6] W. Hao, J. Hang, Y. Tan, W. Su, J. Am. Ceram. Soc. 92, 2937 (2009).

[7] B. Renner, P. Lunkenheimer, M. Schetter, A. Loidl, A. Reller, S.G. Ebbinghaus, J. Appl. Phys. 96, 4400 (2004).

[8] S.G. Ebbinghaus, Prog. Solid State Chem. 35, 421 (2007).

[9] D. Szwagierczak, J. Kulawik, J. Eur. Ceram. Soc. 28, 2075 (2008)

[10] D. Szwagierczak, J. Kulawik, J. Alloys Comp. 491, 465 (2010). 